\title{
Fund Rating Systems and Performance Predictability*
}

\author{
Anne-Sophie Duret Pierre Hereil Philippe Mitaine \\ Nicolas Moussavi Thierry Roncalli
}

April 16, 2008

\begin{abstract}
This paper studies the performance predictability of external fund rating systems. Most investors use 5 stars rated funds to build their portfolios. The underlying idea is that funds which were the best during the last three years will be better performers than the other funds in the future. It implies that the 5 stars rating is a good persistence measure of the performance. Using a Markov modelling and the seminal empirical work of Garnier and Pujol (2007), we show that ratings persistence is poor. It means that fund selection or a fund picking process may not be reduced to choose funds in a 5 stars rated universe.
\end{abstract}

\section{Introduction}

Fund ratings are generally a measure of past performance. However, they are often used by investors in a prospective way. We may ask ourselves if such star rating systems are robust enough to draw some conclusions from an investment point of view.

The paper is organized as follows. In section two, we compar credit rating systems and fund rating systems. We observe a large difference. Contrary to credit rating systems which are qualitative criteria oriented, fund rating systems are generally based on quantitative criteria. In section three, we consider Markov modelling of fund ratings and propose a new measure of the performance persistence. In section four, we explain why the traditional statistical measure of Hurst is not appropriate to measure the persistence of the funds. Section five concludes and gives some implications for fund picking process.

*All the authors are from SGAM Alternative Investments, except Thierry Roncalli who comes both from SGAM Alternative Investments and the University of Evry. 


\section{From credit ratings to fund ratings}

In the credit market, investors need to have an idea of the issuers' creditworthiness. Since the beginning of the twentieth-century, credit rating agencies such as Standard \& Poor's, Moody's or Fitch are specialized in assessing corporations and governments. In order to rate these institutions and their specific issues, credit agencies have developed credit scoring techniques. For example, regarding issuers' credit ratings, «Standard \& Poors Ratings Services employs fundamental credit analysis supplemented by quantitative models». For corporate and financial institutions, the credit analysis especially deals with financial and business risks and follows RAMP (Rating Methodology Profile) systematic framework. The financial analysis consists in evaluating the firm's accounting principles and relies on financial indicators (profitability, leverage, cash flow adequacy, liquidity and financial flexibility) and ratios (profit margins, return on investment, debt/capital, debt/cash flow or debt service coverage, depending on the industry). So as to take into account the risks linked to the firm's environment, the business analysis includes country risk, industry characteristics, competitive position, cost efficiency and profitability to peers. After being determinate, the different variables of these analyzes are combined into a score. But as they are interrelated, their weighting is not fixed and depends on their value.

Besides the credit analysis, the credit agencies have placed emphasis on the qualitative aspect. Actually, it's impossible to reduce the credit rating to a fix and uniform process adapted to each issuer and issue. The factors influencing the credit risk are unique to each industry, issuer and country. That is why they must be weighted case-by-case. After the analytical team reviews information from a variety of sources, the credit rating and the recommendation are determined by the vote of a rating committee. Then, the rating is published and the issuer/issue are placed under surveillance. Depending on the frequency of the information sources, credit rating agencies schedule periodic meetings with the management of the rated company. When major changes occur the analytical team conducts again a comprehensive analysis in order to determine the new rating. Thus, the frequency of the rating isn't rigid and depends on each issue/issuer.

This position is shared by other credit rating agencies. For example, here are some basic principles of Moody's rating system ${ }^{1}$ :

«Quantification is integral to Moody's rating analysis, particularly since it provides an objective and factual starting point for each rating committee's analytical discussion. Those who wish further information on the numerical tools we use may consult our written research on industries and specific issues.

\footnotetext{
${ }^{1}$ source: http://www.moodys.com/moodys/cust/AboutMoodys/AboutMoodys.aspx?topic=rapproach
} 
However, Moody's ratings are not based on a defined set of financial ratios or rigid computer models. Rather, they are the product of a comprehensive analysis of each individual issue and issuer by experienced, well-informed, impartial credit analysts.»

It is very important to note that credit rating is a measure of the likelihood of default. It is then a forward-looking measure.

Given the success of credit rating agencies, other kinds of ratings have been developed such as fund rating. Actually, people who want to invest in mutual funds also need information to choose among the many available funds. The main idea is to distinguish best mutual funds performers from worst. In order to answer this question, agencies like Morningstar but also S\&P have developed since the end of the 90's funds rating methodologies. For instance, the Morningstar rating system (whose first version was introduced in 1985) consists in a stars system. Each fund is assigned a rating from one to five stars, based on its risk-adjusted performance versus an assigned risk peer group. In order to have an efficient information, funds are only compared to others which could be valid substitutes in a diversified portfolio. The funds are classified according to the performance measure : the top $10 \%$ funds are five stars, the next $22.5 \%$ are four stars, etc.

One problem arising with using fund rating systems is that they are purely quantitative based on past performance. But as usually mentioned by disclaimer, "past performance is no indication of future returns". Thus we can ask ourselves about the pertinence of rating systems.

\section{Fund ratings and persistence of the performance}

\subsection{Markov modelling of fund ratings}

A rating system is defined by a set of notches $\mathcal{R}=\left\{\mathcal{R}_{1}, \mathcal{R}_{2}, \ldots, \mathcal{R}_{K}\right\}$. Consider a mutual fund $n$ with rating $\mathcal{R}_{k}$ at time $t$. We note $R_{(n)}(t)=\mathcal{R}_{k}$ where $R_{(n)}(t)$ is the random variable which represents the rating of the mutual fund $n$ at time $t$. Let us define $\pi_{(n)}\left(s, k_{1} ; t, k_{2}\right)$ as the probability that the rating of the mutual fund goes from $\mathcal{R}_{k_{1}}$ at time $s$ to $\mathcal{R}_{k_{2}}$ at time $t$. We have:

$$
\pi_{(n)}\left(s, k_{1} ; t, k_{2}\right)=\operatorname{Pr}\left\{R_{(n)}(t)=\mathcal{R}_{k_{2}} \mid R_{(n)}(s)=\mathcal{R}_{k_{1}}\right\}
$$

This dynamic system is often modelled using an homogeneous Markov chain. Let $\pi=\left(\pi_{i, j}\right)$ be the transition matrix between two consecutive unit periods. This matrix has the properties $\pi_{i, j} \geq 0$ and $\sum_{j=1}^{K} \pi_{i, j}=1$. In this case, we have:

$$
\pi_{(n)}\left(s, k_{1} ; t, k_{2}\right)=\mathbf{e}_{k_{1}}^{\top} \pi^{(t-s)} \mathbf{e}_{k_{2}}
$$


Let us now consider the continuous time $t \in \mathbb{R}_{+}$. We note $\pi(s ; t)$ the transition matrix from time $s$ to time $t$. The transition matrix at period $t$ corresponds to $\pi(t)=\pi(0 ; t)$. We call generator of the transition matrix $\pi(t)$ the matrix $\Lambda=\left(\lambda_{i, j}\right)$ defined by:

$$
\pi(t)=\exp (t \Lambda)
$$

The interpretation of this matrix is the following. If the transition probability from the state $i$ to the state $j(j \neq i)$ in a short elapsed time $\Delta t$ is approximatively proportional to $\Delta t$, we have $\pi(t, i ; t+\Delta t, j)=\lambda_{i, j} \Delta t$. The matrix form is $\pi(t ; t+\Delta t)=\Lambda \Delta t$. It comes that $\pi(t+\Delta t)=\pi(t) \pi(t ; t+\Delta t)=$ $\pi(t) \Lambda \Delta t$ and we have $\mathrm{d} \pi(t)=\pi(t) \Lambda \mathrm{d} t$. Because $\exp (\mathbf{0})$ is equal to the identity matrix, we obtain the solution $\pi(t)=\exp (t \Lambda)$. We may interpret $\lambda_{i, j} \geq 0(j \neq i)$ as the instantaneous transition rate. Moreover, we may show that $\Lambda$ is a Markov generator if it verifies $\sum_{j=1}^{K} \lambda_{i, j}=0$. As the discrete case, we have:

$$
\pi_{(n)}\left(s, k_{1} ; t, k_{2}\right)=\mathbf{e}_{k_{1}}^{\top} e^{\Lambda(t-s)} \mathbf{e}_{k_{2}}
$$

Table 1: Transition matrix of the one month probabilities (in \%) estimated using the $\mathrm{S} \& \mathrm{P}$ fund ratings

\begin{tabular}{c|cccccc}
\hline & $\mathrm{NR}$ & $\star$ & $\star \star$ & $\star \star \star$ & $\star \star \star \star$ & $\star \star \star \star \star$ \\
\hline $\mathrm{NR}$ & 97.14 & 0.41 & 0.62 & 0.54 & 0.77 & 0.52 \\
$\star$ & 1.26 & 84.84 & 13.48 & 0.40 & 0.02 & 0.00 \\
$\star \star$ & 0.78 & 14.23 & 67.30 & 16.96 & 0.73 & 0.00 \\
$\star \star \star$ & 0.70 & 0.84 & 22.34 & 62.30 & 13.75 & 0.07 \\
$\star \star \star \star$ & 0.66 & 0.00 & 0.88 & 15.23 & 76.68 & 6.55 \\
$\star \star \star \star \star$ & 0.38 & 0.00 & 0.00 & 0.05 & 14.70 & 84.87
\end{tabular}

Source : Garnier and Pujol (2007).

Garnier and Pujol (2007) have studied the mutual funds of the equity asset class sold in France from 2000 to 2006. They have estimated the empirical transition matrix of the ratings by the method of maximum likelihood. NR indicate that the fund is not rated. The results for $\mathrm{S} \& \mathrm{P}$ ratings are presented in the Table 1 . We read them in the following way: a mutual fund which is rated 5 stars has a $84.87 \%$ probability to stay 5 stars the next month, but it has also a $14.70 \%$ probability to become 4 stars and a $0.05 \%$ probability to become 3 stars the next month. In order to verify the Markov property of the rating system, we compute the infinitesimal generator with the following 
formula ${ }^{2}$ :

$$
\hat{\Lambda}=12 \ln \hat{\pi}\left(\frac{1}{12}\right)
$$

We obtain:

$$
\hat{\Lambda}=\left(\begin{array}{rrrrrr}
-0.34950 & 0.04757 & 0.07788 & 0.06334 & 0.09558 & 0.06512 \\
0.15957 & -2.16803 & 2.21463 & -0.22631 & 0.02186 & -0.00171 \\
0.09039 & 2.33196 & -5.55237 & 3.31681 & -0.19758 & 0.01080 \\
0.08505 & -0.26478 & 4.36752 & -6.58153 & 2.48664 & -0.09290 \\
0.08169 & 0.02765 & -0.32000 & 2.75972 & -3.54055 & 0.99149 \\
0.04340 & -0.00415 & 0.04254 & -0.25245 & 2.22749 & -2.05683
\end{array}\right)
$$

We remark that some off-diagonal elements are negative. It indicates that the transition matrix of the Table 1 is not Markov. In this case, we may use one of the methods suggested by Israel et al. (2001) to build a valid Markov generator. If we use the method [IRW-1] described in Appendix B, the estimate of the matrix generator is:

$$
\bar{\Lambda}=\left(\begin{array}{rrrrrr}
-0.34950 & 0.04757 & 0.07788 & 0.06334 & 0.09558 & 0.06512 \\
0.15957 & -2.39605 & 2.21463 & 0.00000 & 0.02186 & 0.00000 \\
0.09039 & 2.33196 & -5.74995 & 3.31681 & 0.00000 & 0.01080 \\
0.08505 & 0.00000 & 4.36752 & -6.93921 & 2.48664 & 0.00000 \\
0.08169 & 0.02765 & 0.00000 & 2.75972 & -3.86054 & 0.99149 \\
0.04340 & 0.00000 & 0.04254 & 0.00000 & 2.22749 & -2.31343
\end{array}\right)
$$

\subsection{A new measure of the performance persistence}

Using the previous generator, we may compute all the transition probabilities for all time periods with the formula:

$$
\pi_{(n)}\left(s, k_{1} ; t, k_{2}\right)=\mathbf{e}_{k_{1}}^{\top} e^{\Lambda(t-s)} \mathbf{e}_{k_{2}}
$$

Figures 1, 2 et 3 present some results. The first figure indicates the probability that the mutual fund is rated 5 stars at time $t$ whereas the second figure indicates the probability that the fund is rated 1 star. We remark that traditional order relationships are well verified, in particular:

$$
\operatorname{Pr}\left\{R(t)=5 \star \mid R(0)=\mathcal{R}_{k_{1}}\right\} \geq \operatorname{Pr}\left\{R(t)=5 \star \mid R(0)=\mathcal{R}_{k_{2}}\right\}
$$

if $\mathcal{R}_{k_{1}}>\mathcal{R}_{k_{2}}$ and:

$$
\operatorname{Pr}\left\{R(t)=1 \star \mid R(0)=\mathcal{R}_{k_{1}}\right\} \geq \operatorname{Pr}\left\{R(t)=1 \star \mid R(0)=\mathcal{R}_{k_{2}}\right\}
$$

if $\mathcal{R}_{k_{1}}<\mathcal{R}_{k_{2}}$.

\footnotetext{
${ }^{2}$ All the matrix functions use algorithms based on the Schur decomposition of Appendix
} A. 
Figure 1: Probability $\operatorname{Pr}\left\{R_{(n)}(t)=5 \star \mid R_{(n)}(0)=\mathcal{R}_{k}\right\}$

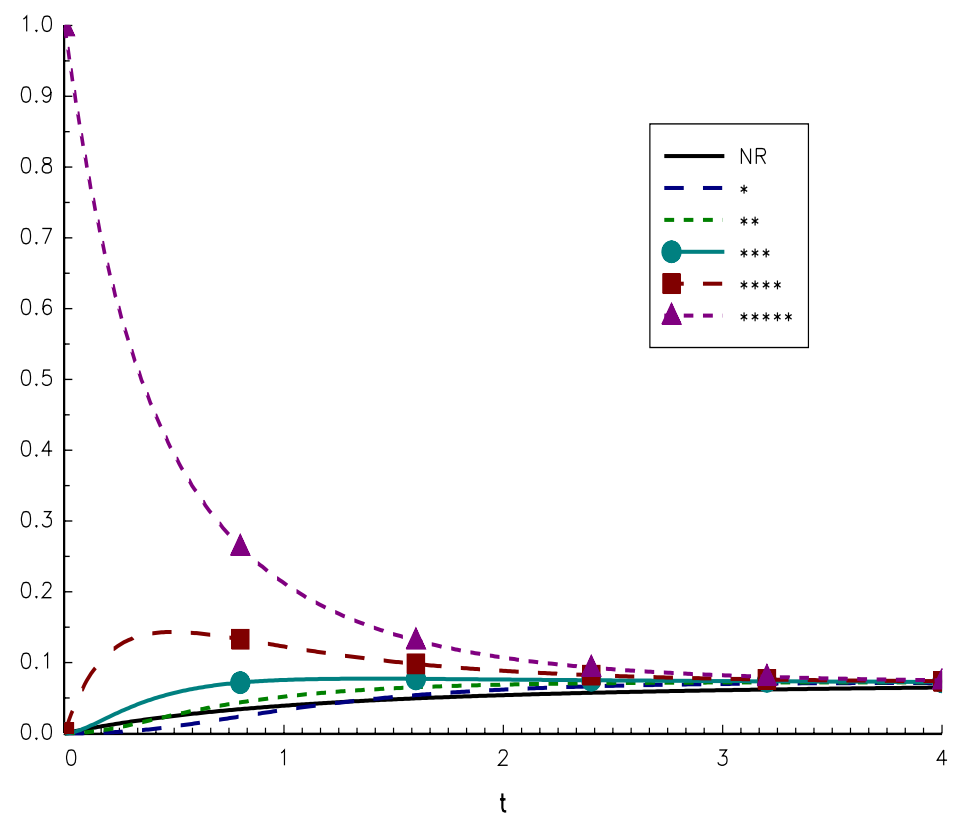

Figure 2: Probability $\operatorname{Pr}\left\{R_{(n)}(t)=1 \star \mid R_{(n)}(0)=\mathcal{R}_{k}\right\}$

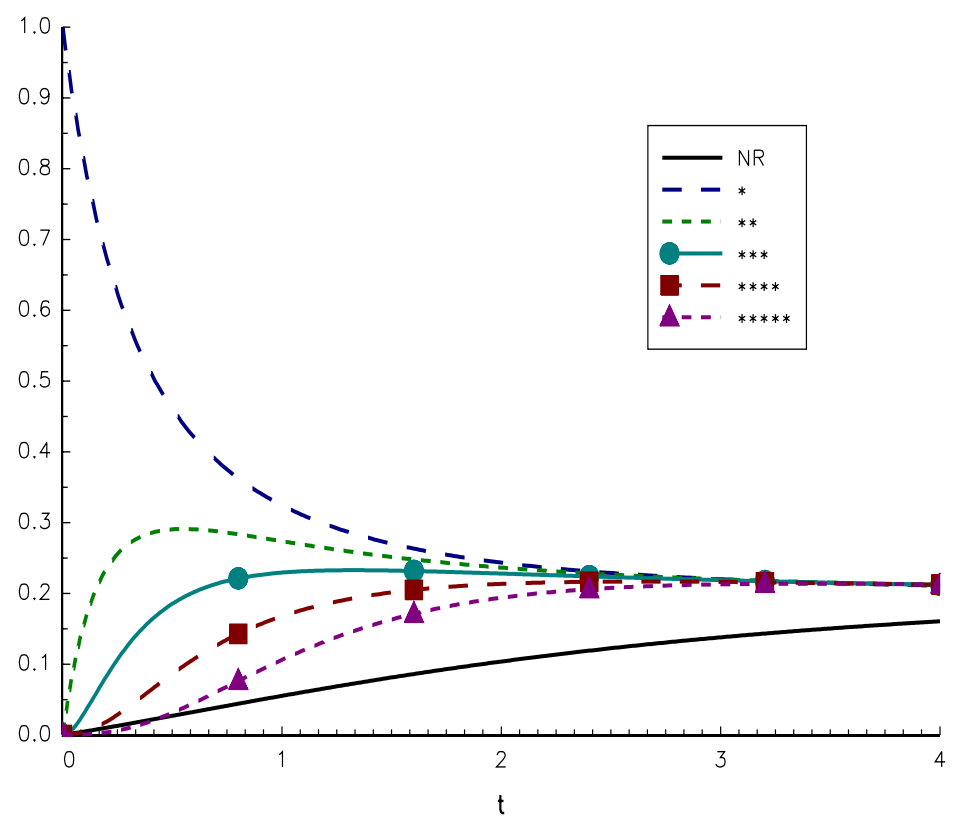


Figure 3: Probability $\operatorname{Pr}\left\{R_{(n)}(t) \geq 3 \star \mid R_{(n)}(0)=\mathcal{R}_{k}\right\}$

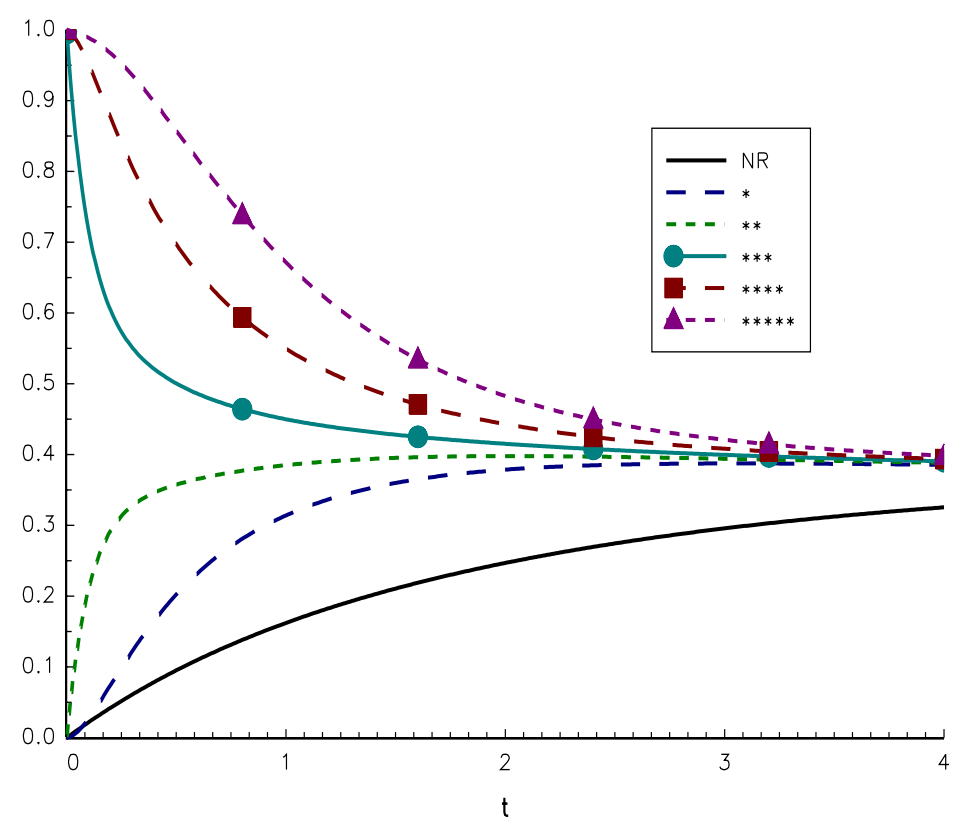

Using the previous generator, we may also compute the two-years transition matrix (probabilities are expressed in \%):

$$
\hat{\pi}(2)=\left(\begin{array}{rrrrrr}
53.72 & 10.38 & 11.22 & 9.38 & 9.91 & 5.39 \\
14.88 & 24.35 & 22.89 & 16.99 & 14.70 & 6.18 \\
13.77 & 23.65 & 22.80 & 17.33 & 15.57 & 6.88 \\
13.13 & 22.81 & 22.55 & 17.55 & 16.37 & 7.60 \\
12.33 & 21.35 & 22.02 & 17.82 & 17.64 & 8.84 \\
11.08 & 19.40 & 21.25 & 18.15 & 19.42 & 10.70
\end{array}\right)
$$

It means that a fund which is rated today 5 stars has a probability of only $10.70 \%$ to stay 5 stars two years later and it has a bigger probability to become one star (this probability is $19.40 \%$ ). Let us define $\bar{t}_{k}$ such that:

$$
\operatorname{Pr}\left\{R(t)=\mathcal{R}_{k} \mid R(0)=\mathcal{R}_{k}\right\}=\operatorname{Pr}\left\{R(t) \neq \mathcal{R}_{k} \mid R(0)=\mathcal{R}_{k}\right\}
$$

If $t \geq \bar{t}_{k}$, a fund rated $\mathcal{R}_{k}$ at time 0 has a bigger probability to have another rating at time $t$. $\bar{t}_{k}$ may be viewed as a persistence measure of the rating $\mathcal{R}_{k}$. If we exclude the state $\mathrm{NR}$, we obtain the following results: $\bar{t}_{k}$ takes respectively the values expressed in months: 5.738, 2.031, 1.522, 2.927 et 4.331 for the ratings one to 5 stars. We remark first that the persistence measure is globally small. Indeed, the most persistent rating is one star, and its persistence does not exceed 6 months. It is interesting to note that the two most persistent ratings are the extreme ratings (one and five stars). 
Figure 4: Persistence probability of the S\&P ratings
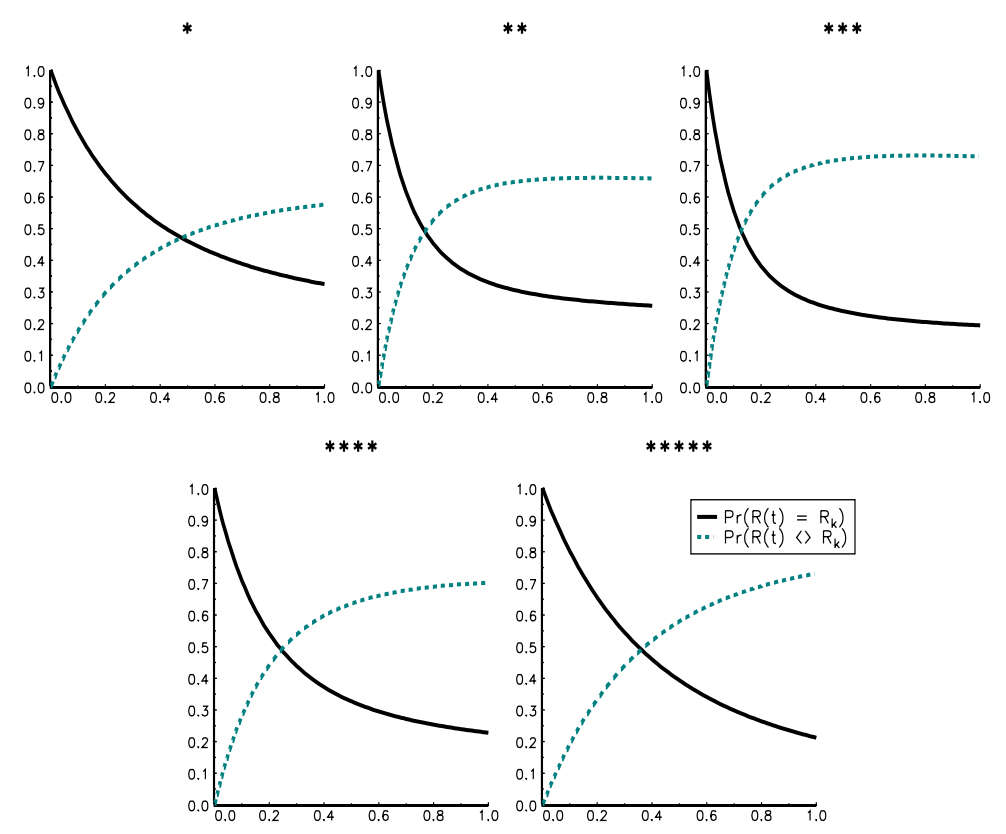

Figure 5: Persistence probability of the Moringstar ratings
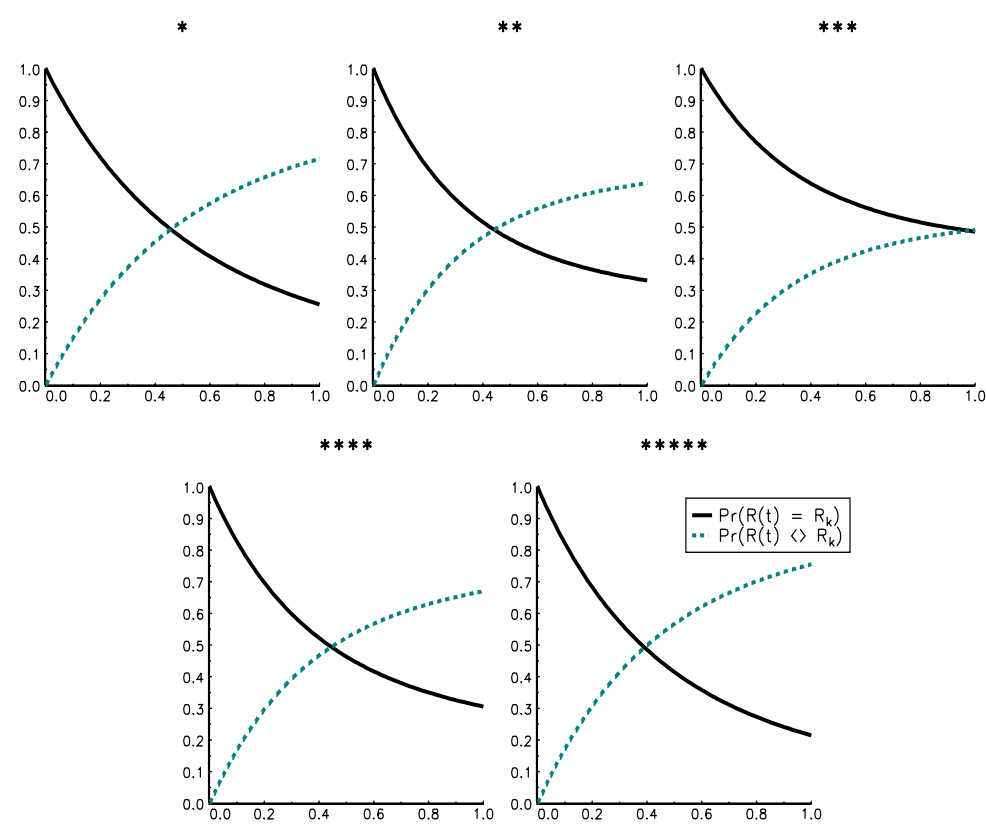
Figure 6: Comparison of persistence probabilities

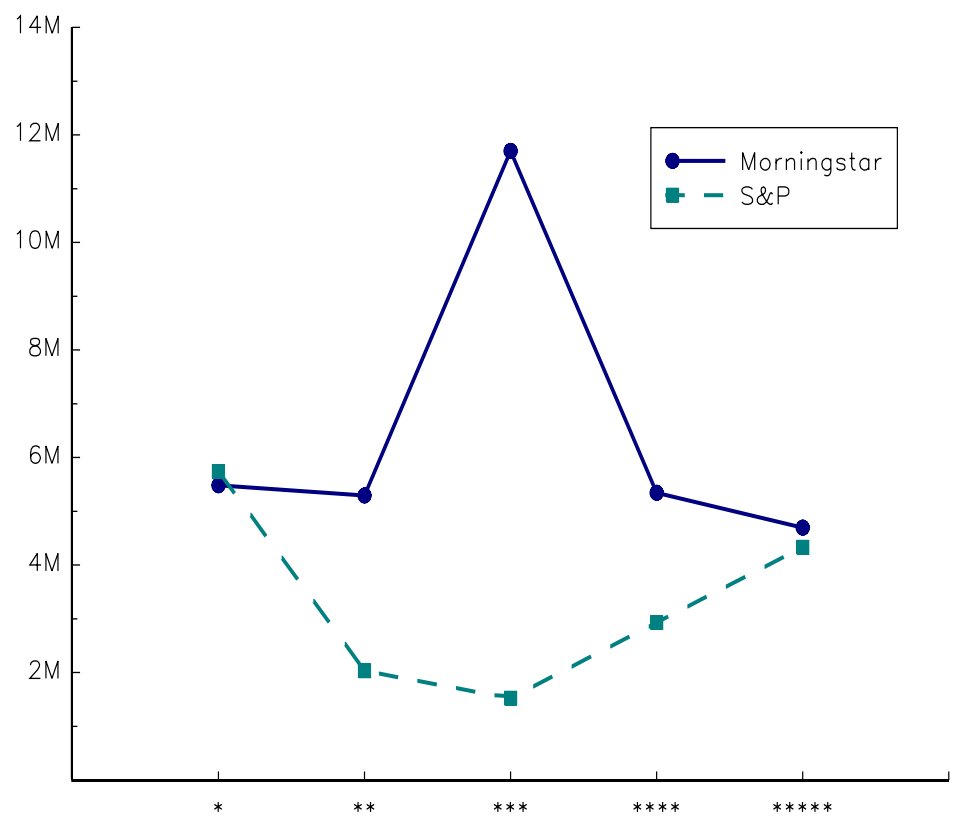

Figure 7: Persistence probabilities of the S\&P Credit ratings

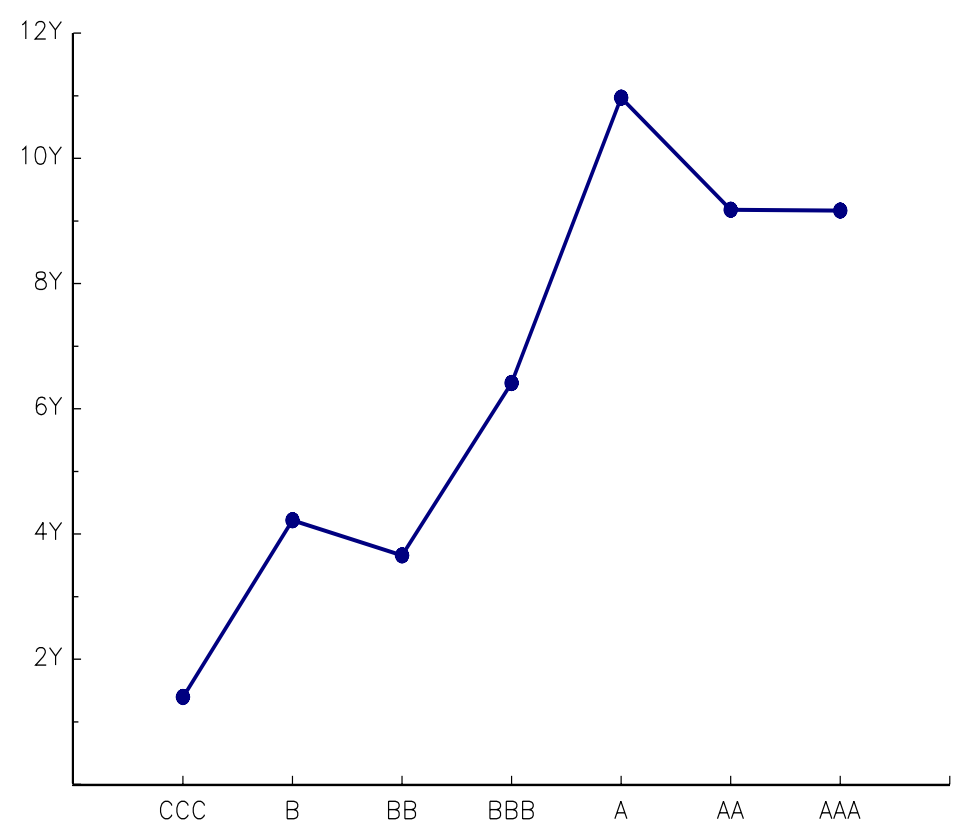


If we use the Morningstar ratings, we obtain Figure 5. It is interesting to compare the persistence measure $\bar{t}_{k}$ of the two rating systems (see Figure 6). We remark that they don't have the same behavior except for the two extreme ratings.

If we compare these persistence measures with those computed in credit ratings systems, they are very different. For S\&P credit ratings ${ }^{3}$, the persistence measure is closed to 9 years for AAA and AA rated companies (see Figure $7)$.

\section{A remark about statistical persistence and Hurst exponent}

\subsection{Definition of long memory processes}

In statistics, the notion of persistence (or long memory) is defined as follows: We say that $X_{t}$ is a long memory process if the associated autocorrelation (positive) function decay at a hyperbolic rate. We may illustrate this phenomena by studying the rate $R_{\rho}(k)$ :

$$
R_{\rho}(k)=\frac{\rho(k+1)}{\rho(k)}
$$

where $\rho(k)$ is the autocorrelation function at horizon $k$. In the case of long memory process, this rate grows and tends to one. It means that the loss of correlation decreases with lags.

\subsection{The Hurst exponent}

In order to characterize the property of persistence, we use generally the statistical measure $H$ called Hurst exponent. This measure may take values between 0 and 1 :

1. statistical persistence corresponds to the case $H>1 / 2$;

2. if $H<1 / 2$, the process is short memory ${ }^{4}$;

3 . if $H$ is equal to $1 / 2$, the process has no memory.

In Figure 8, we have simulated centered processes for several values of $H$. When $H$ is close to 0 , we remark that a positive value is generally followed by a negative value. It is not the case when $H$ is close to 1 . We suppose that these values represent daily returns of a fund. By compounding them, we may then compute the corresponding tracks. We verify that behaviors are very different (see Figure 9). The higher is $H$, the more regular is the fund.

\footnotetext{
${ }^{3}$ We use the transition matrix estimated by Kavvathas (1999).

${ }^{4}$ The decay of the autocorrelation function is geometric.
} 
Figure 8: Simulation of returns for different values of Hurst exponent
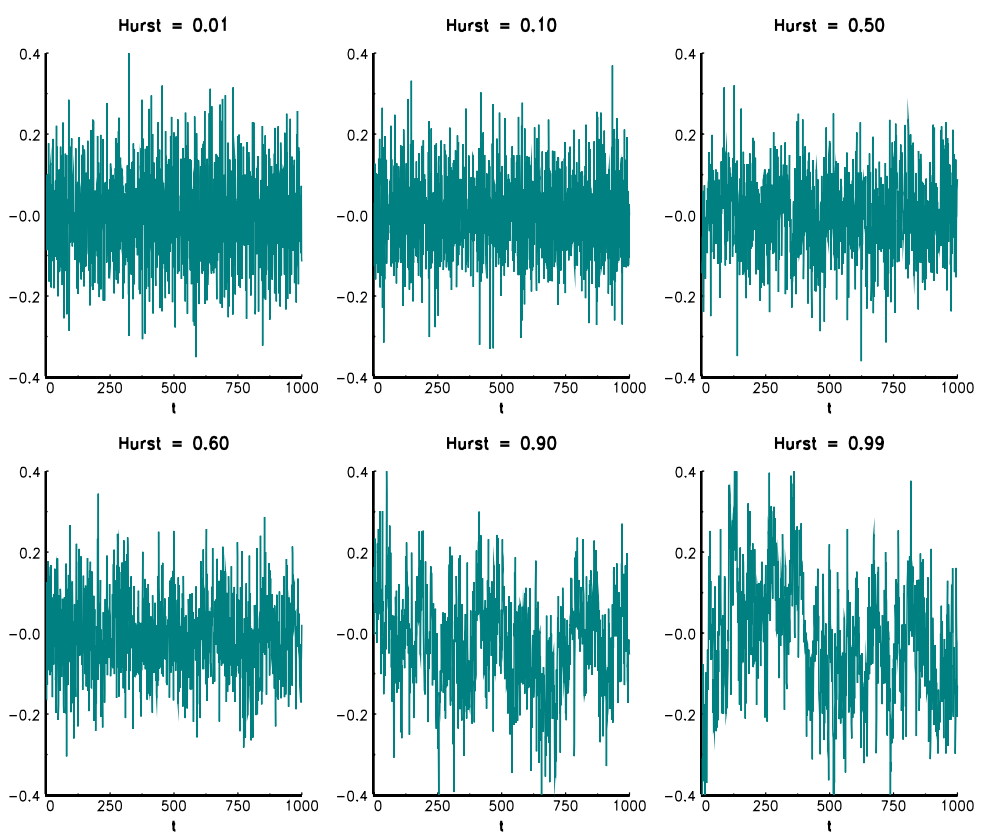

Figure 9: Simulation of tracks
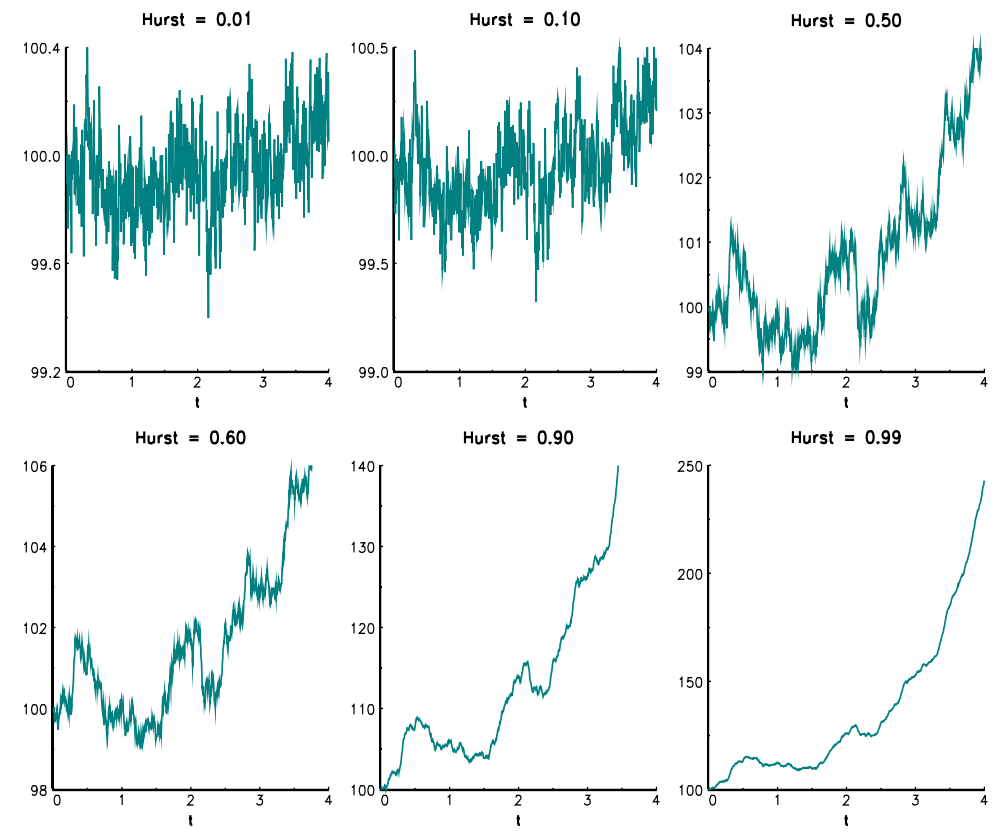


\subsection{Using Hurst exponent to measure the persistence of the performance}

Hurst exponent is used by some fund rating systems (Europerformance Edhec and Lipper). For example, Lipper ranks the funds into three groups $(H<0.45$, $0.45<H<0.55$ and $H>0.55)$ to characterize the rentability persistence. One of the big issue is the estimation of $H$. The most known method is based on R/S analysis. Another method called GPH is based on the spectral density of arfima process. These methods have good properties when the sampling size is very large. However, for small size, the variance of these estimates are huge. It is then difficult to distinguish the case $H>0.5$ for the other cases. Let us consider a Monte Carlo study. The true value of $H$ is 0.7 . We have represented the density of the estimator for different sampling sizes in Figure 10. If the size is equal to 200 , we observe that the estimator has a probability bigger than $20 \%$ to take a value less than 0.5 .

Figure 10: Density of spectral and Hurst estimators

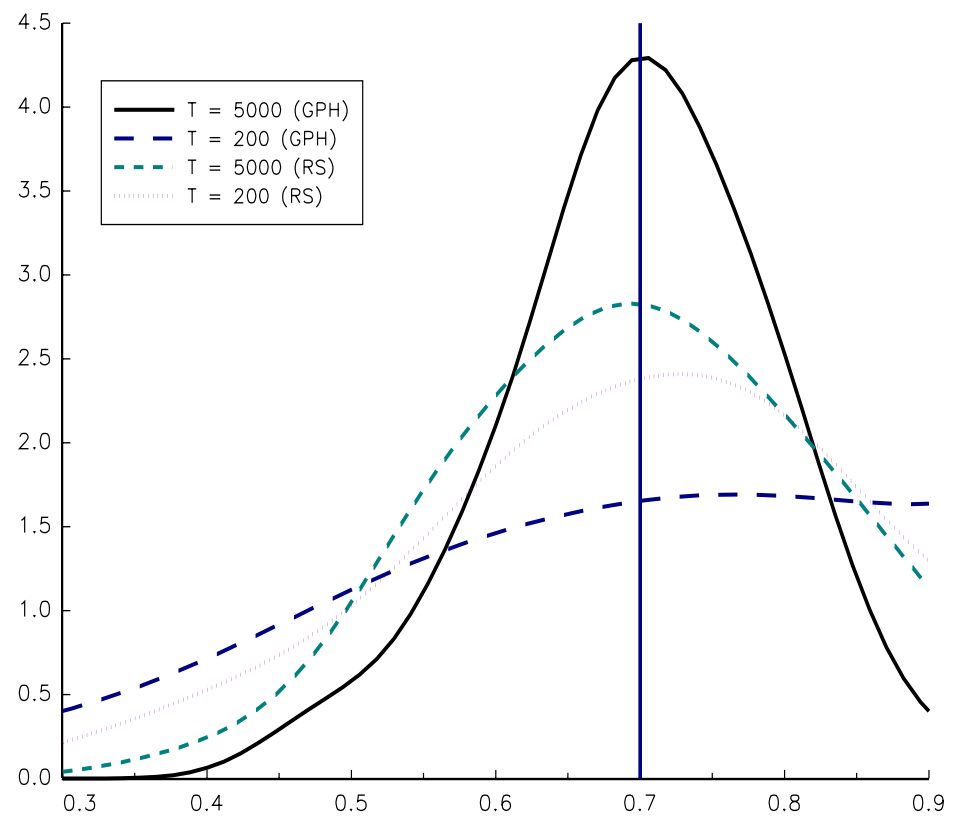

\section{$5 \quad$ Implications for fund picking processes}

We discuss here some implications when one wants to develop an internal fund picking process (for example in the case of multi-management, fund of funds management or structured products on basket of mutual funds). The main message is that fund ratings are only one part of the fund picking process and 
must be completed by qualitative analysis. Moreover, fund picking process make sense if we actively manage the basket of funds and if we implement quick substitution when fund pickers are not comfortable with some funds.

\subsection{Fund rating as a screening tool of mutual fund perfor- mances}

As said previously, fund ratings are a measure of risk-adjusted performances versus an assigned risk peer group. Thus, it enables to reduce the funds universe to the presumed best ones. Actually the number of funds amounts to around 64506 funds at the end of $2006^{5}$. It's impossible to have a thorough knowledge of all these funds. By limiting the analysis to rated funds the investors' work is clearly simplified. But they must not settle for these ranking and only select funds that are best graded. This article has given rise to the fact that fund ratings are non-persistent and the ranking is only based on past performance which doesn't give a good indication of future returns.

That's why fund ratings must be used as a tool to identify potential candidate funds, but fund picking process may not only be reduced to choose the best starred funds.

\subsection{The importance of qualitative analysis}

Given that fund ratings are only based on quantitative models, qualitative analysis are necessary to build a fund picking process. It's the only way of integrating other available information which isn't taken into account by quantitative approach. This covers for example:

1. Team expertise and stability

2. Investment universe

3. Investment philosophy and process

4. Investment bias (style, market cap, sector, etc.)

5. Management style (passive/active, Bottom-up/top-bottom)

This qualitative information is necessary in a fund picking process to understand funds past performances. Actually good rating may be due to biases or lucky bets taken by the manager. Even if the fund performed well last years, it may underperform in the near future. That's why a fund picking process has to assess the adequacy of the management style to the current economic situation and to detect possible flaws in the management process.

\footnotetext{
${ }^{5}$ Investment Company Institute, 2007 Investment Company Fact Book, http: //www.icifactbook.org, 2007.
} 


\subsection{The importance of active management}

It is obvious that a fixed basket of 5 stars funds may not be the good underlying of long maturity product. In asset management, strategic allocation is the core of portfolio construction. Since several years, it is known that strategic allocation may be completed by tactical allocation in order to manage dynamically asset allocation. In order to have exposure to asset classes, the investor may choose between two ways: invest in (passively managed) indexes or in (actively managed) funds. However, as explained before, passive basket of funds (even if all the funds are 5 stars) are not the good solution. It is important to actively manage the underlyings of the basket. It means that switching from one fund to another fund is part of an efficient fund picking process. Fund picking process makes sense if the manager of the basket of funds implements quickly changes decided by fund pickers.

Given that economic situation evolves constantly fund pickers have to regularly update the allocation to optimize the expected risk/return profile according to the current economic prospects. For instance, as we can see in Figure 11, depending on the period it's more pertinent to invest in growth or value funds. A comparison between a smid cap index and a large cap one will give the same result.

Figure 11: One-year performance difference between the Russell 1000 Growth and Value indexes

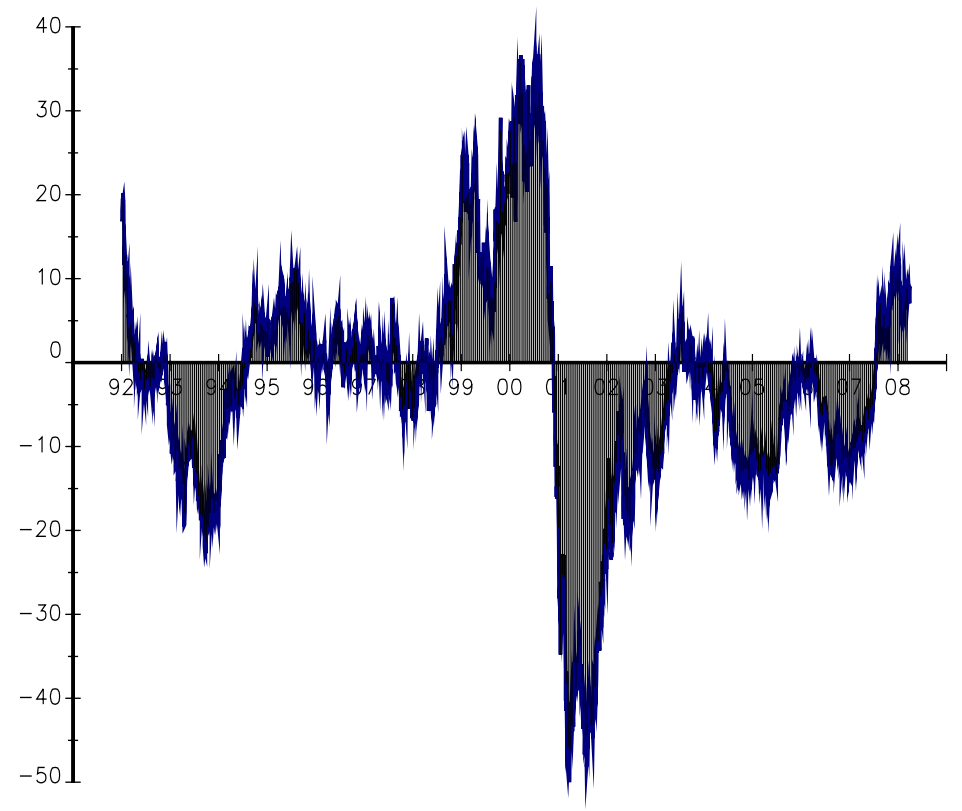




\section{References}

[1] Amenc N. and Le Sourd V., "Rating the Rating", Edhec Risk and Asset Management Research Center, Working Paper, 2005.

[2] Clark A., The Use of Hurst and Effective Return in Investing, Lipper Research Series, May 2003.

[3] Garnier O. and Pujol T., Les étoiles d'aujourd'hui préjugent-elles des étoiles de demain ?, Les Cahiers Scientifiques de l'AMF, 3, March 2007.

[4] Golub G.H. and Van Loan C.F., Matrix Computations, John Hopkins University Press, London, 1989.

[5] Israel R.B., Rosenthal J.S. and Wei J.Z., «Finding Generators for Markov Chains via Empirical Transition Matrices with Applications to Credit Ratings », Mathematical Finance, 11, 2001, p. 245-265.

[6] Kavvathas D., «Estimating Credit Rating Transition Probabilities for Corporate Bonds», University of Chicago, Working paper, 1999.

[7] Morningstar, «The Morningstar Rating Methodology», Morningstar Methodology, March 2005.

[8] Standard \& Poor's, General Description of Corporate 83 Government Rating Credit Rating Methodology, 26 June 2007.

[9] Standard \& Poor's, General Description of the Credit Rating Process, 26 June 2007.

\section{A Schur decomposition and matrix functions}

Let A be a square matrix. The Schur decomposition is:

$$
A=T S T^{*}
$$

with $T$ a unitary matrix ( $T$ is called the transformation matrix) and $S$ a upper diagonal matrix ( $S$ is called the real Schur form).

Let $f$ be a real function defined by:

$$
\begin{aligned}
f: & \mathbb{R} \longrightarrow \mathbb{R} \\
& x \longmapsto y=f(x)
\end{aligned}
$$

It is possible to define this function in the space $\mathbb{M}$ of square matrices:

$$
\begin{aligned}
f: \mathbb{M} & \longrightarrow \mathbb{M} \\
& A \longmapsto B=f(A)
\end{aligned}
$$


Let us consider the case of the square root real function $f(x)=\sqrt{x}=x^{1 / 2}$. If the square matrix $A$ is positive, we may define the matrix $B$ such that:

$$
B B^{*}=B^{*} B=A
$$

$B$ is called the square root of $A$ and we note $B=A^{1 / 2}$.

Let $f$ be a real function. They are several ways to generalize this function in the space of matrices. The most used generalization is based on the Taylor expansion:

$$
f(x)=f(0)+x f^{\prime}(0)+\frac{x^{2}}{2 !} f^{\prime \prime}(0)+\ldots
$$

We may show that if the series converge for $|x|<\alpha$, then the matrix function $f(A)$ defined by:

$$
f(A)=f(0)+A f^{\prime}(0)+\frac{A^{2}}{2 !} f^{\prime \prime}(0)+\ldots
$$

converge to a matrix $B$ if $|A|<\alpha$ and $A$ is a square matrix. We note $B=f(A)$. Let us consider the case of the exponential function. We have:

$$
f(x)=e^{x}=\sum_{k=0}^{\infty} \frac{x^{k}}{k !}
$$

We deduce that the matrix exponential is given by the following expression:

$$
B=e^{A}=\sum_{k=0}^{\infty} \frac{A^{k}}{k !}
$$

Note that the matrix logarithm of $B$ is then the matrix $A$ and we note $A=\ln B$.

Matrix functions are generally computed using the Schur decomposition. In most cases, we may compute $f(A)$ with:

$$
f(A)=T f(S) T^{*}
$$

where $A=T S T^{*}$ is the complex Schur decomposition of $A$. In the general case, we consider the algorithm 11.1.1 of Golub and Van Loan (1989) whereas in the case of the exponential function, we use their algorithm 11.3.1.

\section{B Finding a valid Markov generator}

Let $\hat{\pi}(t)$ be a transition matrix between 0 and $t$. If $\hat{\pi}(t)$ is a Markov matrix, then there exists a generator $\Lambda$ such that:

$$
\hat{\pi}(t)=\exp (t \Lambda)
$$


An estimate of $\Lambda$ is also $\hat{\Lambda}$ defined by:

$$
\hat{\Lambda}=\frac{1}{t} \ln \hat{\pi}(t)
$$

$\hat{\Lambda}$ is a Markov generator if each off-diagonal element is positive and all its rowsums are 0 . If some off-diagonal elements are negative, it means that $\hat{\Lambda}$ is not a Markov generator and $\hat{\pi}(t)$ is not a Markov transition matrix. In order to obtain a valid generator, Israel et al. (2001) suggest two methods by replacing the negative off-diagonal entries with 0 and adding the appropriate value back into other entries to preserve the property of having row-sums 0 .

1. In the first method [IRW-1], we add the negative values back into the corresponding diagonal entry:

$$
\left\{\begin{array}{l}
\bar{\lambda}_{i, j}=\max \left(\hat{\lambda}_{i, j}, 0\right) \quad i \neq j \\
\bar{\lambda}_{i, i}=\hat{\lambda}_{i, i}+\sum_{j \neq i} \min \left(\hat{\lambda}_{i, j}, 0\right)
\end{array}\right.
$$

2. In the second method [IRW-2], we add the negative values back into all the entries of the same row which have the correct sign:

$$
\left\{\begin{array}{l}
G_{i}=\left|\hat{\lambda}_{i, i}\right|+\sum_{j \neq i} \max \left(\hat{\lambda}_{i, j}, 0\right) \\
B_{i}=\sum_{j \neq i} \max \left(-\hat{\lambda}_{i, j}, 0\right) \\
\bar{\lambda}_{i, j}=\left\{\begin{array}{ll}
0 & i \neq j \text { and } \hat{\lambda}_{i, j}<0 \\
\hat{\lambda}_{i, j}-B_{i}\left|\hat{\lambda}_{i, j}\right| / G_{i} \\
\hat{\lambda}_{i, j} & G_{i}=0
\end{array} G_{i}>0\right.
\end{array}\right.
$$

